\title{
Polarization properties of optical metasurfaces of different symmetries
}

\author{
Sergey S. Kruk, ${ }^{1, *}$ Alexander N. Poddubny, ${ }^{2,3}$ David A. Powell, ${ }^{1}$ Christian Helgert, ${ }^{4,1}$ Manuel Decker, ${ }^{1}$ \\ Thomas Pertsch, ${ }^{4}$ Dragomir N. Neshev, ${ }^{1}$ and Yuri S. Kivshar ${ }^{1,2}$ \\ ${ }^{1}$ Nonlinear Physics Center and Center for Ultrahigh Bandwidth Devices for Optical Systems (CUDOS), \\ The Australian National University, Canberra, ACT 0200, Australia \\ ${ }^{2}$ ITMO University, St. Petersburg 199034, Russia \\ ${ }^{3}$ Ioffe Institute of the Russian Academy of Science, St. Petersburg 194021, Russia \\ ${ }^{4}$ Institute of Applied Physics, Abbe Center of Photonics, Friedrich-Schiller-Universität Jena, D-07743 Jena, Germany
}

(Received 21 December 2014; revised manuscript received 17 April 2015; published 5 May 2015)

\begin{abstract}
Optical metasurfaces have become a new paradigm for creating flat optical devices. While being typically an order of magnitude thinner than the wavelength of light, metasurfaces allow control of the phase of propagating light waves across the full $2 \pi$ range and therefore enable the realization of optical elements such as lenses, waveplates, and beam converters. Currently one of the limiting factors of functional metasurfaces is their small range of operational angles. Here we demonstrate both theoretically and experimentally that the angular range can be broadened by increasing the rotational symmetry of metasurfaces. We develop an analytical model based on the discrete dipole approximation that quantitatively describes the response of metasurfaces under oblique excitation. It shows that the effective optical symmetry is doubled for structures with odd rotational symmetry, increasing the angular range correspondingly. We apply and experimentally verify our model for metasurfaces consisting of identical meta-atoms, arranged into square lattices, hexagonal lattices, and on the vertices of a Penrose tiling. The results demonstrate the increasing angular performance with increasing rotational symmetry.
\end{abstract}

DOI: 10.1103/PhysRevB.91.195401

PACS number(s): 78.67.Pt, 42.70.-a

\section{INTRODUCTION}

Metasurfaces are two-dimensional arrangements of individual subwavelength particles made of composite materials. They are typically represented by regular layouts of subwavelength elements - "meta-atoms", which replace the atoms and molecules of conventional materials. Metasurfaces have become a paradigm for the engineering of electromagnetic space and controlling the propagation of waves. As such they are considered as the key elements for the emerging field of metadevices [1], devices with unique and useful functionalities realized by structuring functional matter on the subwavelength scale. Particular interest is attracted by the ability of metasurfaces to abruptly change the phase of light, allowing for polarization and wavefront control. Metasurfaces have recently been demonstrated to operate as flat lenses [2], highly efficient polarization converters [3], vortex beam generators [3], spatial and spectral light modulators [4], etc. The properties of metasurfaces are defined by the response of an individual particle as well as by its coupling to neighbors [5-7].

For many practical applications, metasurfaces are required to operate for a wide range of angles of incident light. As an example, for a metasurface-based lens the range of operating angles is a limiting factor for its numerical aperture and therefore its performance. However, metasurfaces demonstrated to date operate only over a limited range of incident angles. While the angular response of individual meta-atoms has been studied in detail $[8,9]$ the influence of coupling between meta-atoms on the metasurface's angular operation remains largely unexplored and unexploited.

\footnotetext{
*Corresponding author: sergey.kruk@anu.edu.au
}

In most cases, metasurfaces are considered as homogenized media and their effective constitutive parameters are determined via averaging the local fields obtained from a fullwave electromagnetic simulation or analytical calculations [10-13]. The electromagnetic homogenization approach, however, cannot predict the influence of the internal arrangement of the metasurfaces, which has been shown to influence their optical response substantially [14-16].

Here we predict theoretically and demonstrate experimentally that the angular operation of metasurfaces depends strongly on the symmetry of the lattice, similar to crystalline materials whose properties are defined by the type of lattice. Alteration of the lattice symmetry of a metasurface changes the coupling between meta-atoms and therefore changes the optical response. We provide an analytical tool that gives quantitative predictions of the impact of the metasurface point symmetry on its optical properties for any angle of excitation. Our method is based on a coupled-dipole approximation and is applicable to any regular layout of meta-atoms acting as electric dipoles or magnetic dipoles. The method can be generalized for higher-order multipoles. It can be used for any arrangement of meta-atoms with long-range positional order, and therefore it can be applied for both periodic and quasicrystalline structures.

Our quantitative analysis breaks the common perception that symmetry-based analytical methods only provide binary answers as to whether a particular optical phenomenon is allowed or forbidden. As such, our work enables an important extension to the works on point-symmetry group description of individual meta-atoms [17-19], as well as the works on structural chirality [20-23] and helicity [24,25] of optical media.

We apply our method to three types of metasurfaces consisting of identical elements, arranged into fourfold square and sixfold hexagonal lattices, as well as a fivefold Penrose 
tiling. For structures such as the Penrose tiling with odd rotational symmetry, it is shown that the negative lattice vectors also contribute, effectively doubling the structural symmetry for nondiffracted waves. We demonstrate how the angular performance increases with higher rotational symmetry, using circular dichroism as the metric. In our design, circular dichroism is absent for normal illumination of the metasurfaces, and rises at oblique illumination due to the coupling between metaatoms. We calculate analytically and observe experimentally a large suppression of circular dichroism for the Penrose tiling with effective tenfold symmetry, when compared to its sixfold and fourfold counterparts. This suggests that an attractive approach to improve the angular performance of metasurfaces is to increase their rotational symmetry, therefore enabling a wide range of flat metadevices.

\section{SYMMETRY REDUCTION AT THE OBLIQUE INCIDENCE}

We consider a metasurface, illuminated by a plane wave propagating in the positive $z$ direction. We assume that the media are reciprocal, depolarization effects are negligible, and that only the nondiffracted transmitted beam is of interest. The components of the transmitted wave $t$ can be related to those of the incident wave $i$ via the Jones matrix:

$$
\left(\begin{array}{c}
E_{\mathrm{t}, m} \\
E_{\mathrm{t}, n}
\end{array}\right)=\left(\begin{array}{ll}
T_{m m} & T_{m n} \\
T_{n m} & T_{n n}
\end{array}\right)\left(\begin{array}{c}
E_{\mathrm{i}, m} \\
E_{\mathrm{i}, n}
\end{array}\right),
$$

or in compact form $\mathbf{E}_{\mathrm{t}}=\hat{\mathrm{T}} \mathbf{E}_{\mathrm{i}}$. This matrix may be expressed in linear $m, n \in\{\mathrm{TE}, \mathrm{TM}\}$ or circular $m, n \in\{+,-\}$ polarization basis, and conversion between these forms is well known [26]. These two forms of the Jones matrix immediately show which polarization phenomena the media will exhibit, noting that they may be present in one basis but absent in the other. For example, a difference in the amplitude of the diagonal terms leads to circular dichroism $\delta_{\text {circ }}=\left|T_{++}\right|^{2}-\left|T_{--}\right|^{2}$ and linear dichroism $\delta_{\text {lin }}=\left|T_{\mathrm{TE}, \mathrm{TE}}\right|^{2}-\left|T_{\mathrm{TM}, \mathrm{TM}}\right|^{2}$. A difference in amplitude of the cross-terms $\Delta_{\text {circ }}=\left|T_{+-}\right|^{2}-\left|T_{-+}\right|^{2}$ or $\Delta_{\text {lin }}=\left|T_{\mathrm{TE}, \mathrm{TM}}\right|^{2}-\left|T_{\mathrm{TM}, \mathrm{TE}}\right|^{2}$ is known as the asymmetric transmission. A difference in the phase of the diagonal terms in the circular basis $\zeta_{\text {circ }}=\arg \left(T_{++} / T_{--}\right)$is known as circular birefringence, and leads to polarization rotation in the linear basis.

The symmetry properties of Jones matrices are discussed in Ref. [26] for the case of light normally incident upon a meta-surface. However, oblique incidence is another means of spatial symmetry breaking, thus the effective symmetry must be considered for the combined system of a metasurface and an incident wave [8,9]. This is important since metasurfaces may operate for a variety of incident angles, and finite-sized incident beams have a corresponding angular spectrum.

To remove any symmetry reduction effects due to the metasurface's constituent elements, we consider them to be highly symmetric, with infinite rotational symmetry about the $z$ axis, and having mirror symmetry in both the horizontal and vertical planes, with the $D_{\infty h}$ symmetry group. Thus, the effective symmetry is determined purely by the metasurface's lattice arrangement and the incident angle. Since we are interested in the angle-dependent response, we define the rotation and reflection symmetry elements with respect to the wave vector $\mathbf{k}$ and not to the normal to the metasurface plane $z$. In particular, we take into account the following point symmetry elements:

$C_{N}^{(k)}$, rotational axis parallel to the incident wave vector $\mathbf{k}$, where $2 \pi / N$ is the angle of the rotational symmetry;

$\sigma_{v}^{(k)}$, mirror plane, parallel to $\mathbf{k}$;

$i$, center of inversion symmetry.

Note that mirror planes and rotational axes which are neither parallel nor perpendicular to the direction of propagation have no effect on the Jones matrix. We consider four different scenarios of illumination of the two-dimensional metasurface, characterized by angles of illumination $\theta$ and $\phi$, and the presence or absence of a substrate. Table I summarizes corresponding polarization properties.

The symmetry elements of the lattice which dictate the symmetry of the optical response are shown for each case. We calculate the general form of the Jones matrices in both linear and circular bases, following the procedure described in Ref. [26]. From these symmetry rules, we are able to determine permitted polarization phenomena for each case.

Case (i) in Table I corresponds to normal illumination of lattices with rotational symmetry $N>2$. The presence of both $C_{N}^{(k)}$ and $\sigma_{v}^{(k)}$ symmetry elements leads to a diagonal form of the Jones matrix with equal diagonal elements in both linear and circular bases. Accordingly, no polarization phenomena are expected.

Case (ii) in Table I corresponds to two cases: $\sigma_{v}^{(k)}$ symmetry with oblique illumination such that $\mathbf{k}$ is parallel to the symmetry plane; and normal illumination of a rectangular lattice with $N=2$. An important remaining symmetry element in this case is $\sigma_{v}^{(k)}$. As we see from the Jones matrices, in the circular basis cross-polarization is the only phenomenon exhibited. In the linear basis $\delta_{\operatorname{lin}}$ is nonzero, indicating a difference between the transmission of TE and TM polarizations. This is a trivial effect, observable in the majority of natural and artificial materials.

Cases (iii) and (iv) in Table I apply for arbitrary oblique illumination of the lattice. Here mirror planes and rotational axes are not parallel to the direction of illumination, thus neither $C_{N}^{(k)}$ nor $\sigma_{v}^{(k)}$ symmetries exist. An important role is played by the presence or absence of inversion symmetry $i$. This will be satisfied for the case of an optically homogeneous surrounding medium [case (iii)]. The absence of the inversion symmetry $i$ corresponds to the metasurface embedded between two different optical media, as typically occurs for structures fabricated on a substrate.

For case (iii), we can observe from the Jones matrices that the lattice itself contributes to the linear dichroism and circular birefringence. For case (iv), the most general form of the Jones matrix occurs in both linear and circular bases, thus no polarization phenomena are prohibited. This means that dichroism, asymmetric transmission, and birefringence can be observed in these cases, even though they are absent from the structure at normal incidence.

\section{MODEL AND THEORETICAL RESULTS}

In Sec. II we showed how the symmetry of a metasurface is reduced by light at oblique incidence, particularly if symmetry 
TABLE I. (Color online) Symmetry of Jones matrix for different illumination conditions and the relevant symmetry operations.

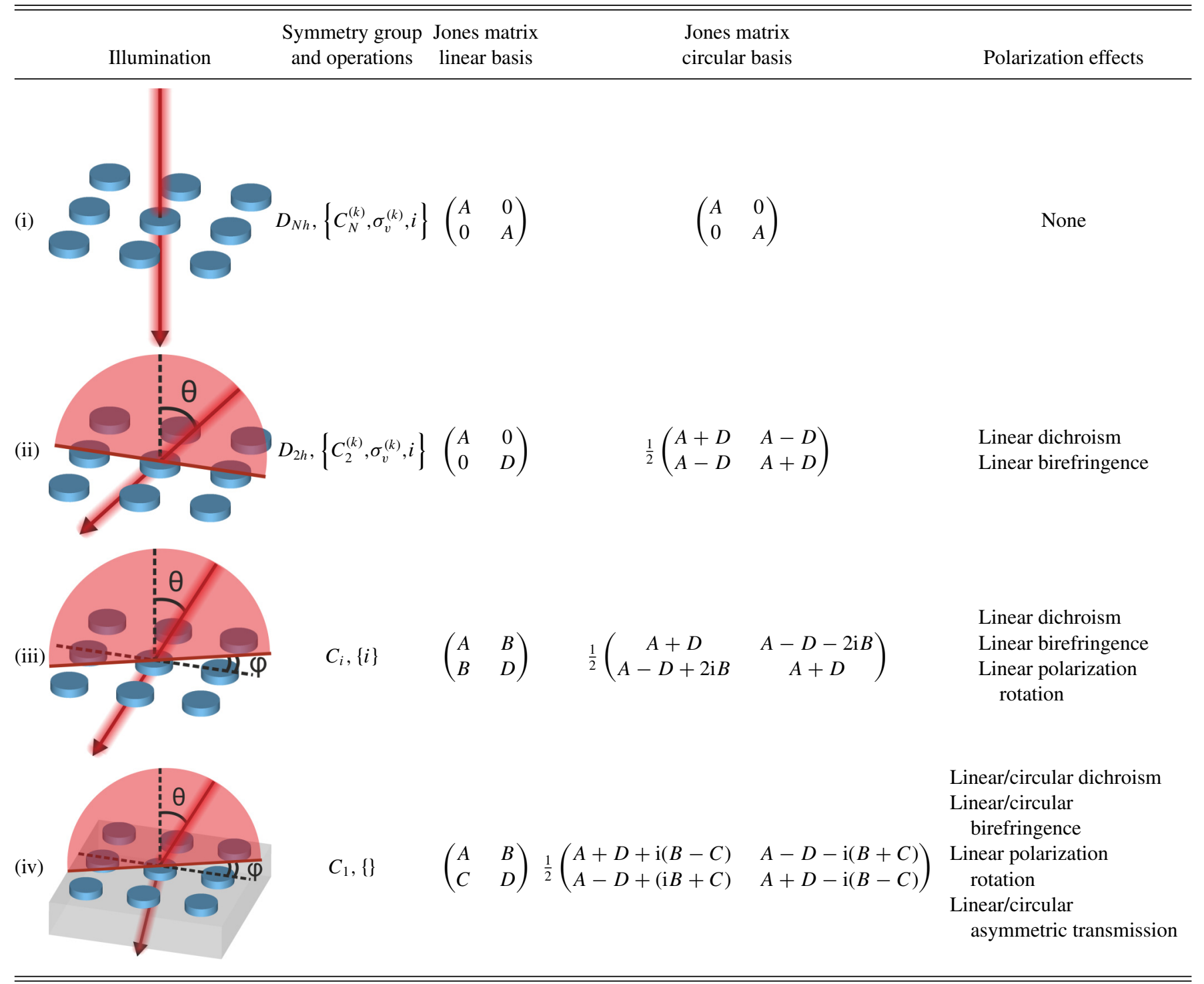

is also broken by the presence of a substrate. In the most general case all polarization phenomena are allowed, however, it is important to define the strength of these effects in different structures. In order to quantitatively study the effect of rotational symmetry on the allowed polarization phenomena, we develop a coupled-dipole microscopic model which describes the polarization-dependent scattering and transmission for metasurfaces with $D_{N h}$ or $C_{N v}$ lattice symmetry. Our theoretical analysis is based on a Fourier expansion. This allows us to use a unified approach for any layout with discrete Fourier spectrum, i.e., with long-range positional order. Thus, the analysis is applicable for both periodic and quasicrystalline arrangements [27].

\section{A. Discrete dipole approximation}

We employ a discrete electric dipole approximation and consider the structure as an array of identical scatterers with polarizability $\hat{\alpha}$, arranged in the $x y$ plane, centered at the vectors $\mathbf{a}$ and embedded in air. The disk symmetry of the individual scatterer means that the polarizability tensor is diagonal and $\alpha_{x x}=\alpha_{y y} \equiv \alpha$. We also neglect the transverse polarizability, $\alpha_{z z}=0$, which is a reasonable assumption for thin disks. In this approximation the structure has the symmetry plane $z=0$ and overall $D_{N h}$ symmetry. We subsequently extend the results to the $C_{N v}$ case by including the effect of the substrate. The equation for the electric field reads

$$
\nabla \times \nabla \times \mathbf{E}=k_{0}^{2} \mathbf{E}+4 \pi \hat{\alpha} \sum_{\mathbf{a}} \mathbf{E}(\mathbf{a}) \delta(\mathbf{r}-\mathbf{a}),
$$

where $k_{0}=\omega / c$. Here and below we use Gaussian-CGS units. We note that our final equations are dimensionless and therefore do not depend on the system of units. The incident wave is characterized by the amplitude $\mathbf{E}_{i}$ and the wave vector k. In order to determine the scattered waves we expand the electric field over the plane wave harmonics, generalizing the approach of Ref. [28] to the case of oblique incidence, $k_{x}, k_{y} \neq 0$ :

$$
\mathbf{E}(\mathbf{r})=\mathbf{E}_{\mathrm{i}} \mathrm{e}^{i \mathbf{k} \cdot \mathbf{r}}+\sum_{\mathbf{G}} \mathbf{E}_{\mathbf{G}} \mathrm{e}^{\mathrm{i}(\mathbf{k}+\mathbf{G}) \cdot \mathbf{r}} .
$$


Substituting Eq. (3) into Eq. (2) and rewriting Eq. (2) in Fourier space along the lines of Ref. [28], we obtain the following system of linear equations for the in-plane components of the electric field:

$$
\mathcal{E}_{\mathbf{G}}=\alpha P\left(k_{\|, G} R\right) \hat{\Pi}_{\mathbf{G}} \sum_{\mathbf{G}^{\prime}} f\left(\mathbf{G}^{\prime}-\mathbf{G}\right) \mathcal{E}_{\mathbf{G}^{\prime}}+\mathcal{E}_{\mathrm{i}} \delta_{\mathbf{G}, 0} .
$$

Here, $\mathbf{G}$ are the two-dimensional diffraction vectors, and $\mathcal{E}_{\mathbf{G}}$ stands for the two-vector $\left(E_{\mathbf{G}, x}, E_{\mathbf{G}, y}\right)^{T}$. The structure factor $f(\mathbf{G})$ is defined by the equation,

$$
f\left(\mathbf{G}^{\prime}-\mathbf{G}\right)=\lim _{\mathcal{N} \rightarrow \infty} \frac{1}{\mathcal{N}} \sum_{j=1}^{\mathcal{N}} e^{\mathrm{i}\left(\mathbf{G}^{\prime}-\mathbf{G}\right) \cdot \mathbf{a}_{j}},
$$

and characterizes the strength of the Bragg diffraction process $\mathbf{k}+\mathbf{G} \rightarrow \mathbf{k}+\mathbf{G}^{\prime}$. The summation in Eq. (5) is performed over all lattice sites $\mathbf{a}_{j}$ with $\mathcal{N}$ being the total number of the sites; the limit for $\mathcal{N} \rightarrow \infty$ is well defined for both crystalline and quasicrystalline lattices [27]. The matrix $\hat{\Pi}_{\mathbf{G}}$ reads

$$
\hat{\Pi}_{\mathbf{G}} \equiv \frac{\mathrm{i}}{S k_{z, G}}\left(\begin{array}{cc}
k_{0}^{2}-k_{x, \mathbf{G}}^{2} & -k_{x, \mathbf{G}} k_{y, \mathbf{G}} \\
-k_{x, \mathbf{G}} k_{y, \mathbf{G}} & k_{0}^{2}-k_{y, \mathbf{G}}^{2}
\end{array}\right),
$$

where $S$ is the mean unit cell size, $\left(k_{x, G}, k_{y, G}\right) \equiv \mathbf{k}_{\|, G}=$ $\left(k_{x}+G_{x}, k_{y}+G_{y}\right), k_{z, G}=\sqrt{k_{0}^{2}-k_{\|}^{2}}$ with $\operatorname{Re}\left\{k_{z, G}\right\}>0$. The interpretation of Eq. (4) is straightforward: The Bragg diffraction leads to coupling of different plane-wave harmonics. The disk form factor,

$$
P\left(k_{\|, G} R\right)=\frac{1}{S} \iint_{\rho<R} d^{2} \rho \mathrm{e}^{-i \mathbf{k}_{\|, G} \cdot \rho}=\frac{2 J_{1}\left(k_{\|, G} R\right)}{k_{\|, G} R},
$$

is introduced as a correction to the point dipole approximation in Eq. (2) [28]; the integration is performed over the disk area. This factor describes suppression of the coupling for waves with the wave vector $k_{\|, G}$ much larger than the inverse disk radius $1 / R$. The transmitted wave is determined from the solution of the linear equation system (4) as

$$
\mathbf{E}_{\mathrm{t}} \equiv \hat{\mathrm{T}} \mathbf{E}_{\mathrm{i}}=e^{i k_{x} x+i k_{y} y+i k_{z, 0} z} \hat{\Pi}_{+} \mathcal{E}_{0},
$$

where $\left[\Pi_{ \pm}\right]_{\alpha \beta}=\delta_{\alpha \beta}-\kappa_{ \pm, \alpha} \kappa_{ \pm, \beta} / k_{0}^{2}$ and $\kappa_{ \pm}=\left(k_{x}, k_{y}, \pm k_{z}\right)$.

Equations (4) and (7) are applicable for arbitrary periodic and quasicrystalline structures characterized by a discrete Fourier spectrum. Our goal is to obtain analytical expressions for the Jones matrix elements in the lowest nonvanishing order in the polarizability $\alpha$. Microscopically, the polarization-dependent effects, such as circular dichroism and asymmetric transmission, stem from the coupling to the waves with $\mathbf{G} \neq 0$. Instead of solving the infinite system Eq. (4) numerically, we keep only one set of the nonzero vectors $\mathbf{G}$ with the same length, that has the lattice symmetry and provides the main contribution to the polarization effect. The optimal value of $G=|\mathbf{G}|$, maximizing the coupling parameter $|f(\mathbf{G}) P(G R)|$ in Eq. (4), is on the order of the inverse spacing $2 \pi / a$ between the neighboring nanodisks. For larger $G$ the coupling is suppressed due to the disk form factor $P\left(k_{\|, G} R\right)$; for smaller $G$ it is quenched due to the vanishing structure factor $f(\mathbf{G})$. Thus, for even rotational degree of the lattice $N$, we include only the vector $\mathbf{G}=0$ and $N$ diffraction vectors $\mathbf{G}_{n}=G\left(\cos \phi_{n}, \sin \phi_{n}, 0\right)^{T}, n=1 \ldots N, \phi_{n}=2 \pi(n-1) / N$. We also retain only the diffractive coupling between the vectors $\mathbf{G}_{n}$ and the vector $\mathbf{G}=0$. Hence, due to the rotational symmetry, taking only the structure factor component $f \equiv f\left(\mathbf{G}_{n}\right)$ is sufficient to describe the Bragg diffraction. In the case of the odd rotational degree $N$ we also include $N$ diffraction vectors $-\mathbf{G}_{n}$ opposite to the vectors $\mathbf{G}_{n}$; their coupling to the wave with $\mathbf{G}=0$ is described by the complex conjugated structure factor $f^{*} \equiv f\left(-\mathbf{G}_{n}\right)$.

Now, the infinite system of Eq. (4) is reduced to $2(N+1)$ or $2(2 N+1)$ linear equations for even and odd $N$, respectively. In particular, for even $N$ these equations read

$$
\begin{aligned}
\mathcal{E}_{0} & =\alpha f^{*} \hat{\Pi}_{0} \sum_{n=1}^{N} \mathcal{E}_{\mathbf{G}_{\mathbf{n}}}+\mathcal{E}_{\mathrm{i}}, \\
\mathcal{E}_{\mathbf{G}_{n}} & =\alpha P(G R) f \hat{\Pi}_{\mathbf{G}_{\mathbf{n}}} \mathcal{E}_{0}, \quad n=1 \ldots N,
\end{aligned}
$$

and similarly for odd $N$ :

$$
\begin{aligned}
\mathcal{E}_{0} & =\alpha \hat{\Pi}_{0} \sum_{n=1}^{N}\left(f^{*} \mathcal{E}_{\mathbf{G}_{\mathbf{n}}}+f \mathcal{E}_{-\mathbf{G}_{\mathbf{n}}}\right)+\mathcal{E}_{\mathrm{i}}, \\
\mathcal{E}_{\mathbf{G}_{n}} & =\alpha P(G R) f \hat{\Pi}_{\mathbf{G}_{\mathbf{n}}} \mathcal{E}_{0}, \quad n=1 \ldots N, \\
\mathcal{E}_{-\mathbf{G}_{n}} & =\alpha P(G R) f^{*} \hat{\Pi}_{-\mathbf{G}_{\mathbf{n}}} \mathcal{E}_{0} .
\end{aligned}
$$

Here, we have replaced $P\left(k_{\|, 0} R\right) \equiv P(\omega R / c)$ by unity and $P\left(k_{\|, G_{n}} R\right)$ by $P(G R)$, since the disk size and the interdisk spacing are much smaller than the wavelength of light. An important advantage of our reduced Fourier space approach over the direct real space techniques is the possibility to use the same set of equations for all considered crystalline and quasicrystalline systems. The only difference between the square, hexagonal, and Penrose lattices encoded in Eqs. (8) and (9) is the rotational symmetry degree $N$ and the characteristic structure factor value $f$.

\section{B. Analytical expressions for the Jones matrices}

Solving Eqs. (9) and (8) in the lowest order in $\alpha$ and $k_{0} / G$ and substituting the result in Eq. (7) we obtain

$$
\hat{\mathrm{T}}=\left(\begin{array}{cc}
1+U+V \cos \tilde{N} \phi & V \sin \tilde{N} \phi \\
V \sin \tilde{N} \phi & 1+U \cos ^{2} \theta-V \cos \tilde{N} \phi
\end{array}\right)
$$

where $\tilde{N}=N$ for even $N$ and $\tilde{N}=2 N$ for odd $N$. Here,

$$
\begin{aligned}
U & =i \alpha k_{0}^{2} / S, \\
V(\theta) & =-\tilde{N} \cos \theta \frac{k_{0}}{G} P(G R)|f|^{2} U^{\tilde{N}-1} \sin ^{\tilde{N}-2} \theta .
\end{aligned}
$$

The matrix is presented in the basis of TE and TM polarized waves.

In order to generalize the result Eq. (10) to the case of $C_{N v}$ symmetry we take into consideration the absence of the symmetry plane $z=0$. Microscopically, the reduction of the symmetry has two independent origins, (i) asymmetric scatterers [see Fig. 1(a)] and (ii) the presence of a substrate. Analytically, the presence of the substrate can be accounted for by multiplying the Jones matrix Eq. (10) with the transfer matrix of a homogeneous substrate:

$$
\hat{\mathrm{T}}\left(C_{N v}\right)=\left(\begin{array}{cc}
t_{\mathrm{TE}} & 0 \\
0 & t_{\mathrm{TM}}
\end{array}\right) \hat{\mathrm{T}}\left(D_{N h}\right),
$$


(a) meta-atom

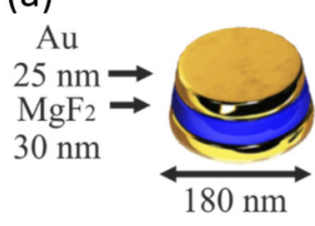

(b) $\tilde{\mathrm{N}}=4$
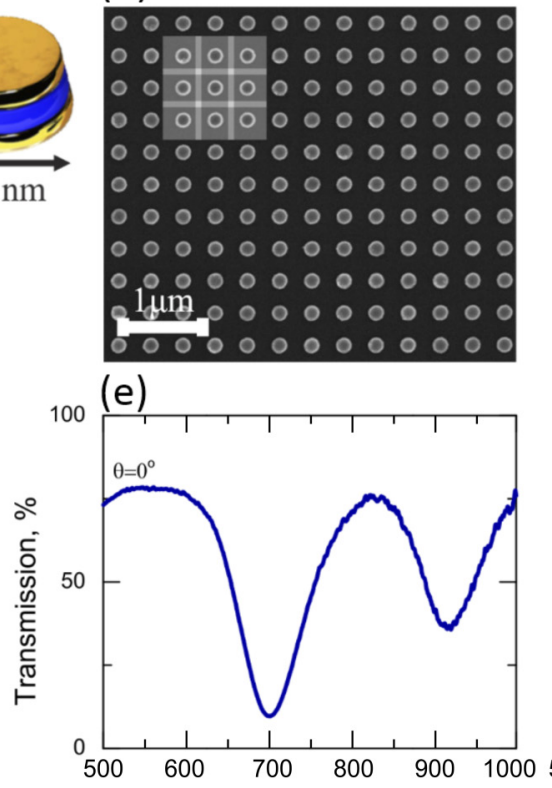

(c) $\tilde{\mathrm{N}}=6$

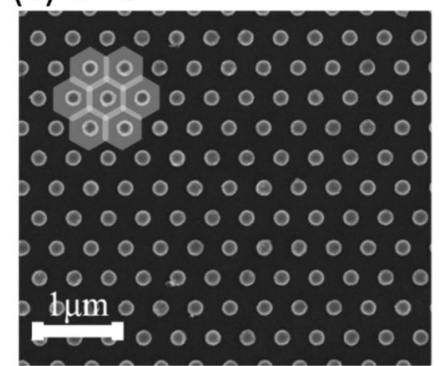

(f)

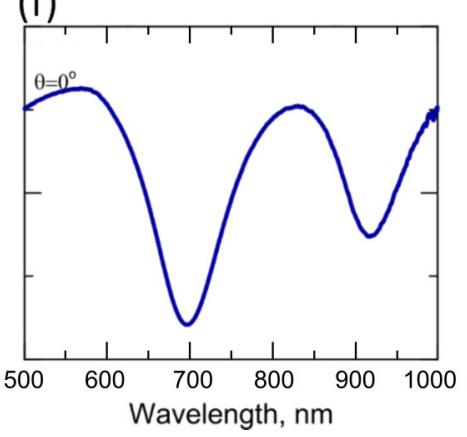

(d) $\widetilde{N}=10$

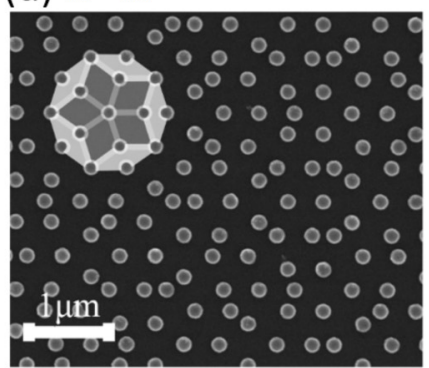

(g)

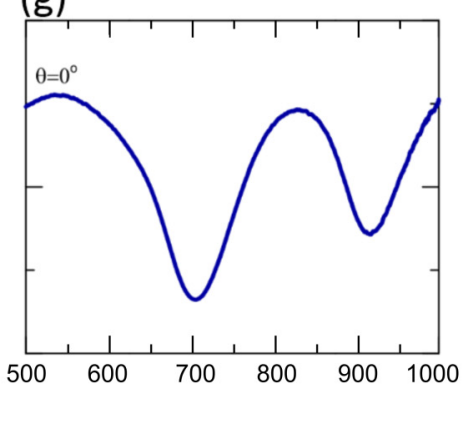

FIG. 1. (Color online) Fabricated metasurfaces and their transmission spectra. (a) A single nanoparticle, (b)-(d) scanning electron micrographs of three fabricated metasurfaces with $\tilde{N}=4,6$, and 10, respectively, (e)-(g) transmission spectra of the corresponding metasurfaces at normal incidence.

where $t_{\mathrm{TE}}$ and $t_{\mathrm{TM}}$ are the substrate transmission coefficients for the corresponding polarizations, $t_{\mathrm{TE}}-t_{\mathrm{TM}} \sim \Delta_{\mathrm{sub}} \sin ^{2} \theta$ for small $\theta$. The approximation mediated by Eq. (12) does not take into account the possible modification of the coupling between the disks due to the presence of the substrate. However, it captures the symmetry of the problem and provides estimated values and the functional dependence for the observed effects. Combining Eq. (10) and Eq. (12) we obtain the following expressions for the components of the transmission matrix in the circular basis:

$$
\begin{gathered}
T_{ \pm, \pm}=\frac{1}{2}\left(\tilde{t}_{\mathrm{TE}}+\tilde{t}_{\mathrm{TM}}\right) \pm \frac{\mathrm{i}}{2} V\left(t_{\mathrm{TM}}-t_{\mathrm{TE}}\right) \sin \tilde{N} \phi, \\
T_{ \pm, \mp}=\frac{1}{2}\left(\tilde{t}_{\mathrm{TM}}-\tilde{t}_{\mathrm{TE}}\right) \mp \frac{\mathrm{i}}{2} V\left(t_{\mathrm{TM}}-t_{\mathrm{TE}}\right) \sin \tilde{N} \phi, \\
\tilde{t}_{\mathrm{TE}}=t_{\mathrm{TE}}(1+U+V \cos \tilde{N} \phi), \\
\tilde{t}_{\mathrm{TM}}=t_{\mathrm{TM}}\left(1+U \cos ^{2} \theta-V \cos \tilde{N} \phi\right) .
\end{gathered}
$$

Equations (10)-(16) give a quantitative form of the Jones matrices represented in Table I, fully accounting for the symmetry of the problem and describing the associated polarization phenomena in metasurfaces both on a substrate $\left(C_{N v}\right.$ symmetry group) and embedded into a homogeneous medium ( $D_{N h}$ symmetry group). They provide explicit expressions for the dependence of the transmitted specular waves on the polar $(\theta)$ and azimuthal $(\phi)$ angles of the incident wave vector $\boldsymbol{k}$.

\section{Analytical expressions for the polarization phenomena}

We next derive explicit expressions for the two polarization phenomena which are of greatest interest: linear polarization rotation and circular dichroism. The linear polarization rotation effect is observed already for the $D_{N h}$ symmetry. The rotation angles for two polarizations are given by

$$
\chi_{\mathrm{TE}}=\arctan \operatorname{Re} \frac{C}{A}, \quad \chi_{\mathrm{TM}}=\arctan \operatorname{Re} \frac{B}{D},
$$

and are expressed in terms of the transmission matrix components in Table I. Substituting the explicit values for the elements of the matrix Eq. (10) we obtain in the lowest order in $V$,

$$
\begin{aligned}
& \chi_{\mathrm{TE}}=\arctan \left(v_{\mathrm{TE}} \cos \theta \sin ^{\tilde{N}-2} \theta \cos \tilde{N} \phi\right), \\
& \chi_{\mathrm{TM}}=\arctan \left(v_{\mathrm{TM}}(\theta) \cos \theta \sin ^{\tilde{N}-2} \theta \cos \tilde{N} \phi\right),
\end{aligned}
$$

with

$$
\begin{aligned}
v_{\mathrm{TE}} & =-\frac{\tilde{N} k_{0} P(G R)|f|^{2}}{G} \operatorname{Re} \frac{U^{\tilde{N}-1}}{1+U}, \\
v_{\mathrm{TM}}(\theta) & =-\frac{\tilde{N} k_{0} P(G R)|f|^{2}}{G} \operatorname{Re} \frac{U^{\tilde{N}-1}}{1+U \cos ^{2} \theta},
\end{aligned}
$$

for the structure embedded in the homogeneous medium.

The second effect, circular dichroism, requires further reduction of the structure symmetry, i.e., the absence of the horizontal mirror symmetry plane. The general expression for the circular dichroism strength in the linear order in $V$ is found from Eq. (13) as

$$
\delta_{\text {circ }}=-\sin \tilde{N} \phi \operatorname{Im}\left[\left(\tilde{t}_{\mathrm{TE}}^{*}+\tilde{t}_{\mathrm{TM}}^{*}\right) V\left(t_{\mathrm{TM}}-t_{\mathrm{TE}}\right)\right] .
$$

Equation (20) demonstrates that the circular dichroism in our model depends on the linear polarization rotation (the factor $V$ ) and on the linear birefringence in the substrate (the factor $\left.t_{\mathrm{TM}}-t_{\mathrm{TE}}\right)$. Approximating $t_{\mathrm{TM}}-t_{\mathrm{TE}}$ as $\Delta_{\mathrm{sub}} \sin ^{2} \theta$ and introducing the transmission coefficient $t=\left(\tilde{t}_{\mathrm{TE}}+\tilde{t}_{\mathrm{TM}}\right) / 2$ we 
rewrite Eq. (20) in the final form,

$$
\delta_{\text {circ }}=W \cos \theta \sin ^{\tilde{N}} \theta \sin \tilde{N} \phi,
$$

where $\theta$ and $\phi$ are the polar and azimuthal angles of illumination and the parameter,

$$
W=\operatorname{Im}\left[\frac{\tilde{N} k_{0}}{G} P(G R)|f|^{2} U^{\tilde{N}-1} t^{*} \Delta_{\text {sub }}\right],
$$

characterizes the strength of the effect.

Equations (18) and (21) give quantitative predictions of linear polarization rotation and circular dichroism for the nondiffracted transmitted beam in lattices of different symmetry. The appearance of the parameter $\tilde{N}$ in these formulas effectively doubles the degree of symmetry for lattices with odd-order rotational symmetry. This can be interpreted as an additional symmetry introduced by the $\pi$ rotational degeneracy of the polarization vector.

\section{EXPERIMENTAL VERIFICATION}

Next, we verify experimentally our general analytical theory and results. For this purpose, we fabricate three types of optical metasurfaces with different symmetries $N=4,6,5$ (and, respectively, $\tilde{N}=4,6$, and 10). The metasurfaces are composed of a regular two-dimensional lattice of meta-atoms, created by metal-dielectric-metal nanodisks [see Fig. 1(a)]. For each meta-atom, we use two metal disks separated by a dielectric spacer. This enables us to obtain two distinct resonant responses of the meta-atom to the incident light at two different wavelengths. Each meta-atom possesses two strong resonances corresponding to the excitation of an electric dipole and a magnetic dipole in the metal-dielectric-metal meta-atom $[29,30]$. The rotationally symmetric shape of the individual nanoparticles ensures that the circular dichroism of our samples depends solely on the symmetry of the lattice.

The fabrication is performed by using electron-beam lithography on a glass substrate, covered with a conducting, 10 -nm thin layer of indium tin oxide (ITO). The lithography is followed by evaporation of layers of gold/magnesium fluoride $\left(\mathrm{MgF}_{2}\right) /$ gold and a sequential lift-off procedure in acetone. The ITO layer is used to prevent charging effects during electron-beam lithography. The Penrose tiling is generated by the de Bruijn method [31]. The size of the patterned area is
$100 \mu \mathrm{m} \times 100 \mu \mathrm{m}$. The surface density of the nanoparticles is kept identical at one nanoparticle per $0.16 \mu \mathrm{m}^{2}$ for all three types of metasurfaces, to allow direct comparison of the experimental results. For a square lattice, this corresponds to a lattice period of $400 \mathrm{~nm}$ in both lateral directions. This fabrication procedure leads to a tapered form of individual nanoparticles [Fig. 1(a)] with a taper angle of approximately $10^{\circ}$. While both the tapering and the presence of the substrate break the symmetry in the direction perpendicular to the metasurfaces, we have verified by the full-wave numerical simulations that the influence of tapering on the symmetry breaking is much less than the effect of the substrate. Figures 1(b)-1(d) show the fabricated metasurfaces.

First, we measure the transmission spectra of all metasurfaces at normal incidence. The measurements are performed using a halogen lamp light source and an Ocean-Optics spectrometer. The spectra are shown in Figs. 1(e)-1(g). We observe that the metasurfaces exhibit two resonant responses in the wavelength region between $0.5 \mu \mathrm{m}$ and $1 \mu \mathrm{m}$, associated with the electric dipole and magnetic dipole resonances, respectively [15].

We next proceed to oblique spectroscopic measurements in the linear polarization basis. According to Table I the presence of nontrivial polarization phenomena corresponds to the appearance of nonzero off-diagonal components of the Jones matrices in the linear basis [cases (iii) and (iv)]. We experimentally define the ratio of the components $|C| /|A|$ and $|B| /|D|$ by measuring the transmission of cross- and copolarized components of the incident linearly polarized light: $\sqrt{\frac{I_{\mathrm{TE}, \mathrm{TM}}}{I_{\mathrm{TE}, \mathrm{TE}}}}$ and $\sqrt{\frac{I_{\mathrm{TM}, \mathrm{TE}}}{I_{\mathrm{TM}, \mathrm{TM}}}}$. The corresponding spectra are shown in Fig. 2. We see that for the case $\sin (\tilde{N} \phi)=0$ [case (ii) in Table I] the values of the off-diagonal components remain close to zero, and can be attributed to imperfections of fabrication and experiment. For the case $\sin (\tilde{N} \phi) \approx 1$ [case (iv) in Table I] the off-diagonal components are high for the fourfold rotationally symmetric metasurface, have lower but significant values for the sixfold symmetric metasurfaces, and are barely detectable for the Penrose metasurface with $\tilde{N}=10$. As the effect is much less pronounced for the 10-fold quasicrystalline metasurface, the measurements were performed at higher incident angle. We also notice that for the fourfold sample the maximum of the effect is observed for the $\sin (4 \phi)$ value of 0.93 rather than the expected value of 1 . The presence of off-diagonal
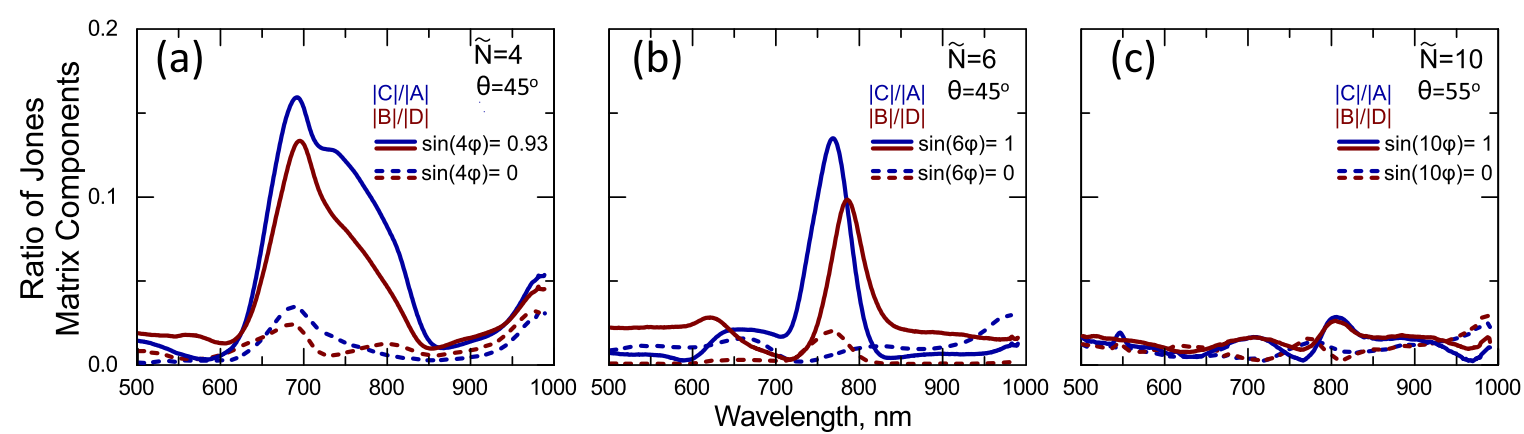

FIG. 2. (Color online) Ratio of off-diagonal and diagonal components of linearly polarized Jones matrices at oblique illumination for the metasurfaces with $\tilde{N}=4,6$, and 10. Blue lines correspond to TE-polarized incident light. Red lines correspond to TM-polarized incident light. Solid lines correspond to case (iv) illumination (see Table I for details) with $\sin \tilde{N} \phi \approx 1$. Dashed lines correspond to case (ii) illumination with $\sin \tilde{N} \phi \approx 0$. 

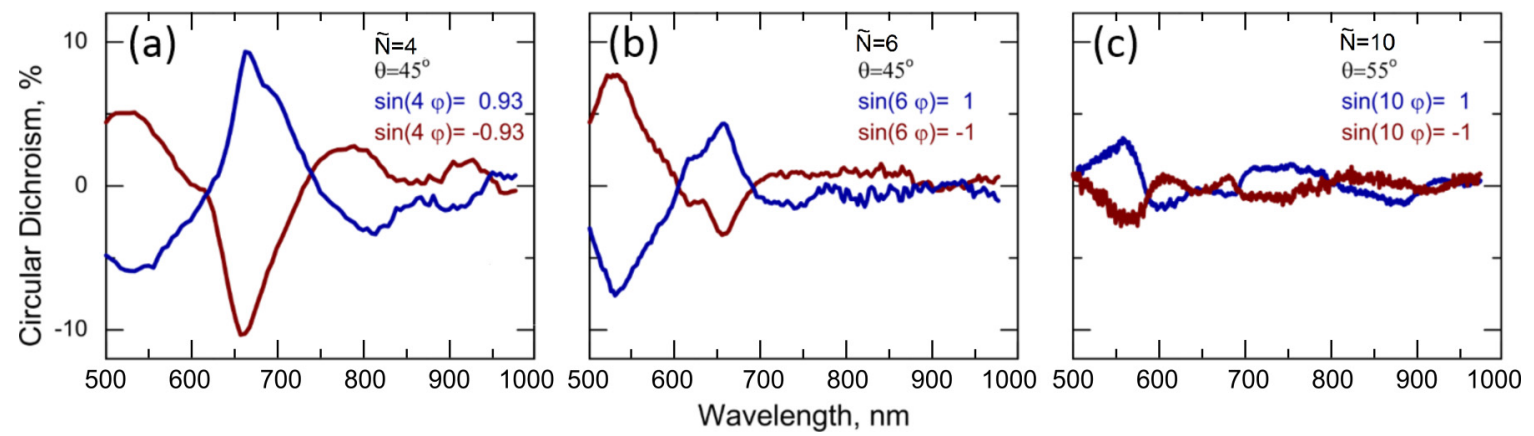

FIG. 3. (Color online) Circular dichroism spectra at the oblique illumination for the metasurfaces with $\tilde{N}=4,6$, and 10 . Blue and red lines correspond to circular dichroism measured for opposite values of $\sin \tilde{N} \phi$.

components suggests that the metasurfaces should exhibit a number of polarization effects at oblique incidence.

We further focus on one particular polarization phenomenon, circular dichroism described by Eq. (21), and study its dependence versus three physical parameters: $\tilde{N}, \theta$, and $\phi$. We use the same white-light transmission spectroscopy setup to measure the circular dichroism as $\delta_{\text {circ }}=I_{++}-I_{--}$, where $I_{++}$and $I_{--}$correspond to the transmission intensities of the right- and left-polarized light. Examples of the measured spectra of circular dichroism are shown in Fig. 3. We observe that the highest values of the circular dichroism are observed for the metasurface with the lowest rotational symmetry, as predicted by the theory and resulting Eq. (21). Blue and red curves correspond to circular dichroism, measured for opposite values of $\sin \tilde{N} \phi$. In accordance with Eq. (21), the value of the dichroism changes to opposite as $\sin \tilde{N} \phi$ changes its sign. We next proceed to a quantitative comparison of circular dichroism measured experimentally and predicted with Eq. (21). In the experiment we notice that changes of incident angle of light lead to small shifts of spectral position of resonances. To remove the influence of these spectral shifts, we average the dichroism value within a spectral region containing both resonances, between $0.5 \mu \mathrm{m}$ and $1 \mu \mathrm{m}$ wavelength using the following equation,

$$
\delta_{\text {circ }}^{\text {ave }}=\left(\lambda_{\max }-\lambda_{\text {min }}\right)^{-1}\left|\int_{\lambda_{\min }}^{\lambda_{\max }} \delta_{\text {circ }}(\lambda) d \lambda\right| .
$$

Figure 4 shows the circular dichroism average as a function of angles $\phi$ and $\theta$ for the three types of metasurfaces. The dots correspond to experimental measurements and the
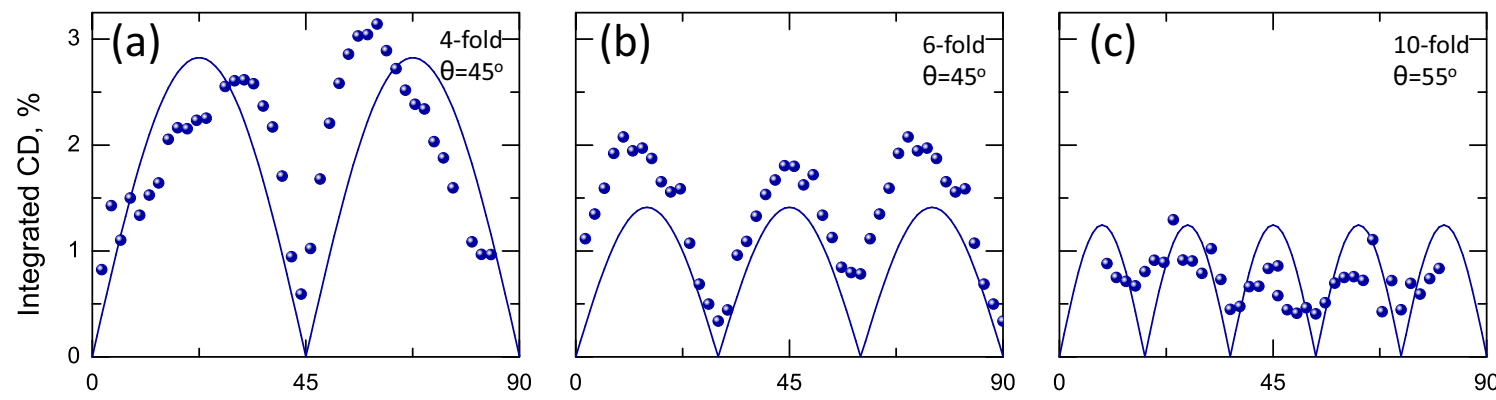

$\varphi$ angle, degrees
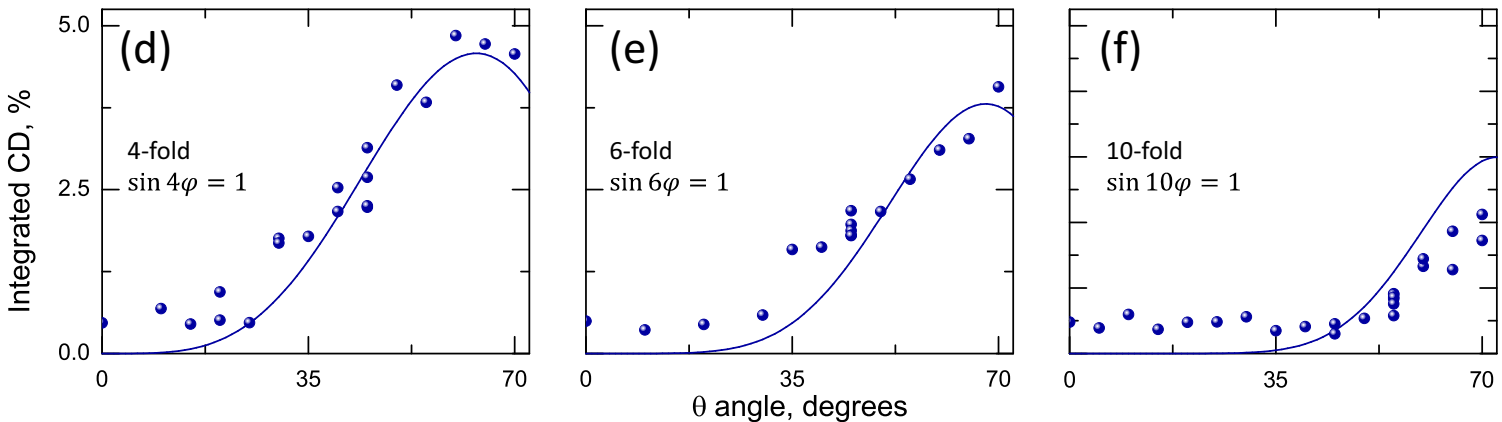

FIG. 4. (Color online) Summary of results for averaged circular dichroism $\delta_{\text {circ }}^{\text {ave }}$ Dots correspond to experimental measurements; lines correspond to analytical prediction from Eq. (21) with $W=0.16$. (a)-(c) The circular dichroism as a function of the azimuthal angle $\phi$, while (d)-(f) show the circular dichroism as a function of the polar angle $\theta$. 
solid lines are the analytical curves as per Eq. (21) with $W=0.16$. We observe that the experimental data follow well the theoretically predicted dependencies. In addition, we check the agreement between experiment and theory for different intervals of spectral averaging and confirm that the agreement is preserved for the intervals comparable or larger than the width of a resonance. The dichroism amplitude is also in a good quantitative agreement with Eq. (22). In particular, for the square lattice with $\tilde{N}=4$, the lattice spacing $a$ is equal to $400 \mathrm{~nm}$ and $|f|^{2}=1$. Since the transmission dip in the experimental spectra of Fig. 1 almost reaches $0 \%$, we conclude that $|U| \sim 1$. The transmission coefficient through the glass substrate is close to unity. The substrate-induced TE-TM splitting $\Delta_{\text {sub }}$ can be estimated from the Fresnel formulas. The result yields $\left|\Delta_{\text {sub }}\right|=4\left|n_{\text {glass }}-1\right|^{2} /\left|n_{\text {glass }}+1\right|^{2} \approx 0.1$, where $n_{\text {glass }} \approx 1.45$ is the glass refractive index and the multiple reflections in the substrate are neglected. We also assume that the factor $P(G R)$ in Eq. (22) is close to unity. This leads to the estimation,

$$
|W| \sim \frac{4 \tilde{N} a}{\lambda} \frac{\left|n_{\text {glass }}-1\right|^{2}}{\left|n_{\text {glass }}+1\right|^{2}} \approx 0.2,
$$

in the vicinity of the electric dipole resonance at $\lambda=700 \mathrm{~nm}$, in agreement with the spectral-averaged experimental value of 0.16 .

These experimental results demonstrate that our analytical model quantitatively captures all the key features of circular dichroism at oblique incidence. This verifies the reduced angular sensitivity for structures with higher rotational symmetry. For the Penrose structure with effective 10-fold symmetry, the circular dichroism is entirely negligible for polar angles below $50^{\circ}$, whereas for the fourfold and sixfold symmetry lattices it is significant over this angle range.

\section{CONCLUSIONS}

By employing the general results of a symmetry analysis, we have studied the effect of the metasurface symmetries on their polarization properties. We have presented a general approach based on the discrete-dipole approximation that provides unambiguous tools for analyzing the impact of the inherent symmetry of the metamaterial lattice on their far-field response. The method is applicable to any ordered layout of meta-atoms acting as electric dipoles or magnetic dipoles, and it can be generalized for higher-order multipoles. More specifically, we have studied experimentally the polarization effects in metasurfaces consisting of elements arranged into square lattices, hexagonal lattices, and on the vertices of a Penrose tiling, and have compared the results with our general theoretical model. Our findings allow quantification of the impact of interelement coupling and lattice symmetry on the optical properties of metamaterials, particularly the circular dichroism at oblique incidence. This contribution is separated from the response associated with individual isolated meta-atoms. We have shown how the optical activity, circular dichroism, and asymmetric transmission depend on both the lattice symmetry and the angle of incidence. Our results demonstrate the influence of symmetry of the metasurfaces' layouts on coupling conditions between individual elements and hence their collective optical response. This makes symmetry of an inner arrangement an important degree of freedom in metasurface design. We believe our results constitute a step forward in the design of optically isotropic metasurfaces and open new possibilities for wide-angle metadevices.

\section{ACKNOWLEDGMENTS}

The authors acknowledge both financial and facility support from the Australian Research Council (ARC), the Federal Ministry of Education and Research (PhoNa, Germany), Go8DAAD joint research cooperation scheme, and the Thuringian State Government (MeMa, Germany). C.H. gratefully acknowledges a postdoctoral fellowship from the German Academic Exchange Service (DAAD). A.P. has been supported by Russian Science Foundation Grant No. 14-12-01067. The authors acknowledge the help of F. Karouta, K. Vora, and J. Tian from the Australian National Fabrication Facility.
[1] N. I. Zheludev and Y. S. Kivshar, From metamaterials to metadevices, Nat. Mater. 11, 917 (2012).

[2] F. Aieta, P. Genevet, M. A. Kats, N. Yu, R. Blanchard, Z. Gaburro, and F. Capasso, Aberration-free ultrathin flat lenses and axicons at telecom wavelengths based on plasmonic metasurfaces, Nano Lett. 12, 4932 (2012).

[3] Y. Yang, W. Wang, P. Moitra, I. I. Kravchenko, D. P. Briggs, and J. Valentine, Dielectric meta-reflectarray for broadband linear polarization conversion and optical vortex generation, Nano Lett. 14, 1394 (2014).

[4] B. Walther, C. Helgert, C. Rockstuhl, F. Setzpfandt, F. Eilenberger, E.-B. Kley, F. Lederer, A. Tünnermann, and T. Pertsch, Spatial and spectral light shaping with metamaterials, Adv. Mater. 24, 6300 (2012).

[5] N. Liu, S. Kaiser, and H. Giessen, Magnetoinductive and electroinductive coupling in plasmonic metamaterial molecules, Adv. Mater. 20, 4521 (2008).
[6] M. Decker, S. Linden, and M. Wegener, Coupling effects in low-symmetry planar split-ring resonator arrays, Opt. Lett. 34, 1579 (2009).

[7] M. Decker, N. Feth, C. M. Soukoulis, S. Linden, and M. Wegener, Retarded long-range interaction in splitring-resonator square arrays, Phys. Rev. B 84, 085416 (2011).

[8] E. Plum, X.-X. Liu, V. A. Fedotov, Y. Chen, D. P. Tsai, and N. I. Zheludev, Metamaterials: Optical activity without chirality, Phys. Rev. Lett. 102, 113902 (2009).

[9] S. A. Mousavi, E. Plum, J. Shi, and N. I. Zheludev, Coherent control of birefringence and optical activity, Appl. Phys. Lett. 105, 011906 (2014).

[10] J. V. Kranendonk and J. Sipe, in Foundations of the Macroscopic Electromagnetic Theory of Dielectric Media, edited by E. Wolf (Elsevier, Amsterdam, 1977), pp. 245-350. 
[11] A. Sihvola, Electromagnetic Mixing Formulas and Applications, IEE Publication Series (Institution of Electrical Engineers, London, 1999).

[12] G. Milton, The Theory of Composites, Cambridge Monographs on Applied and Computational Mathematics (Cambridge University Press, Cambridge, 2002).

[13] S. Tretyakov, Analytical Modeling in Applied Electromagnetics, Artech House Electromagnetic Analysis Series (Artech House, London, 2003).

[14] C. Helgert, C. Rockstuhl, C. Etrich, C. Menzel, E.-B. Kley, A. Tünnermann, F. Lederer, and T. Pertsch, Effective properties of amorphous metamaterials, Phys. Rev. B 79, 233107 (2009).

[15] S. S. Kruk, C. Helgert, M. Decker, I. Staude, C. Menzel, C. Etrich, C. Rockstuhl, C. Jagadish, T. Pertsch, D. N. Neshev, and Y. S. Kivshar, Optical metamaterials with quasicrystalline symmetry: Symmetry-induced optical isotropy, Phys. Rev. B 88, 201404(R) (2013).

[16] M. Albooyeh, S. Kruk, C. Menzel, C. Helgert, M. Kroll, A. Krysinski, M. Decker, D. N. Neshev, T. Pertsch, C. Etrich, C. Rockstuhl, S. A. Tretyakov, C. R. Simovski, and Y. S. Kivshar, Resonant metasurfaces at oblique incidence: interplay of order and disorder, Sci. Rep. 4, 4484 (2014).

[17] W. J. Padilla, Group theoretical description of artificial electromagnetic metamaterials, Opt. Express 15, 1639 (2007).

[18] J. D. Baena, L. Jelinek, and R. Marqués, Towards a systematic design of isotropic bulk magnetic metamaterials using the cubic point groups of symmetry, Phys. Rev. B 76, 245115 (2007).

[19] C. M. Bingham, H. Tao, X. Liu, R. D. Averitt, X. Zhang, and W. J. Padilla, Planar wallpaper group metamaterials for novel terahertz applications, Opt. Express 16, 18565 (2008).
[20] K. Matra and N. Wongkasem, Left-handed chiral isotropic metamaterials: analysis and detailed numerical study, J. Opt. A: Pure Appl. Opt. 11, 074011 (2009).

[21] O. Isik and K. P. Esselle, Analysis of spiral metamaterials by use of group theory, Metamaterials 3, 33 (2009).

[22] C. M. Reinke, T. M. De la Mata Luque, M. F. Su, M. B. Sinclair, and I. El-Kady, Group-theory approach to tailored electromagnetic properties of metamaterials: An inverse-problem solution, Phys. Rev. E 83, 066603 (2011).

[23] T. Cao, C. Wei, L. Mao, and Y. Li, Extrinsic 2D chirality: giant circular conversion dichroism from a metal-dielectric-metal square array, Sci. Rep. 4, 7442 (2014).

[24] I. Fernandez-Corbaton, Forward and backward helicity scattering coefficients for systems with discrete rotational symmetry, Opt. Express 21, 29885 (2013).

[25] I. Fernandez-Corbaton, X. Vidal, N. Tischler, and G. MolinaTerriza, Necessary symmetry conditions for the rotation of light, J. Chem. Phys. 138, 214311 (2013).

[26] C. Menzel, C. Rockstuhl, and F. Lederer, Advanced Jones calculus for the classification of periodic metamaterials, Phys. Rev. A 82, 053811 (2010).

[27] C. Janot, Quasicrystals. A Primer (Clarendon Press, Oxford, 1994).

[28] A. N. Poddubny, Wood anomalies in resonant photonic quasicrystals, Phys. Rev. B 83, 075106 (2011).

[29] G. Dolling, C. Enkrich, M. Wegener, J. F. Zhou, C. M. Soukoulis, and S. Linden, Cut-wire pairs and plate pairs as magnetic atoms for optical metamaterials, Opt. Lett. 30, 3198 (2005).

[30] V. M. Shalaev, W. Cai, U. K. Chettiar, H.-K. Yuan, A. K. Sarychev, V. P. Drachev, and A. V. Kildishev, Negative index of refraction in optical metamaterials, Opt. Lett. 30, 3356 (2005).

[31] N. De Bruijn, Algebraic theory of Penrose's non-periodic tilings of the plane, Proc. Ser. A 84, 39 (1981). 